\title{
Pneumonia por Varicela Associada com Síndrome da Angústia Respiratória Aguda. Relato de Dois Casos*
}

\author{
Varicella Pneumonia Complicated with Acute \\ Respiratory Distress Syndrome. Two Cases Report
}

Marcelo Moreno ${ }^{1}$, Ricardo Castelão', Susana Orrico Peres², Suzana Margareth Lobo ${ }^{3}$.

\section{RESUMO}

JUSTIFICATIVA E OBJETIVOS: A varicela é uma doença exantemática causada pela infecção primária do vírus varicela zoster (VVZ). A pneumonia pelo WVZ complicada com a síndrome da angústia respiratória aguda (SARA) é rara e associa-se a altas taxas de morbimortalidade. $O$ objetivo deste estudo foi apresentar dois casos de pneumonia por varicela que evoluíram com SARA e outras disfunções orgânicas.

RELATO DOS CASOS: Paciente de 15 anos, imunocomprometido com a síndrome da imunodeficiência adquirida (SIDA) e uma paciente do sexo feminino imunocompetente, foram admitidos na UTI com quadro clínico de varicela, SARA, trombocitopenia e acidose graves. Além disso, disfunção cardiovascular e falência renal ocorreram no primeiro e segundo casos, respectivamente. Foram tratados com aciclovir além de ventilação mecânica protetora.

CONCLUSÕES: Os dois casos de pneumonia por varicela, que apresentaram SARA e disfunções de múltiplos órgãos, obtiveram boa evolução clínica.

1. Residente em Medicina Intensiva na FAMERP.

2. Professora Adjunta da Disciplina de Clinica Médica da FAMERP. Doutora em Medicina pela Universidade de São Paulo.

3. Professora Adjunta da Disciplina de Clinica Médica da FAMERP. Coordenadora do Serviço de Terapia Intensiva e da Residência Medica em Medicina Intensiva. Doutora em Medicina pela Universidade de São Paulo.

${ }^{*}$ Recebido da Unidade de Terapia Intensiva da Faculdade de Medicina de São José do Rio Preto (FAMERP), São José do Rio Preto, SP

Apresentado em 25 de novembro de 2006

Aceito para publicação em 27 de fevereiro de 2007

Endereço para correspondência:

Prof. Dra Suzana Margareth Lobo

Av. Brigadeiro Faria Lima, 5544

Faculdade de Medicina de São José do Rio Preto

15090-000 São José do Rio Preto, SP

E-mail: utigeral.hbase@famerp.br

(C)Associação de Medicina Intensiva Brasileira, 2007
Unitermos: Disfunções de Múltiplos Órgãos, pneumonia, varicela, SARA, SIDA

\section{SUMMARY}

BACKGROUNG AND OBJECTIVES: Varicella is an exantematic disease caused by varicella-zoster virus. Varicella pneumonia complicated with acute respiratory distress syndrome (ARDS) is very rare in adults and is associated with high morbimortality. We report two cases of ARDS secondary to varicella-zoster virus pneumonia.

CASES REPORT: We report two cases of ARDS and multiple organ dysfunction syndrome (MODS) secondary to varicella-zoster virus pneumonia. A 15-year-old man with human immunodeficiency virus (HIV) infection and a 29-year-old immunocompetent female were admitted in the ICU with primary varicella infection and pneumonia. Both cases progressed towards ARDS, severe thrombocytopenia and acidosis. In addition cardiovascular and renal failure occurred in the first and second patients, respectively. Treatment consisted of immediate administration of intravenous acyclovir and a lung-protective ventilation strategy.

CONCLUSIONS: Both cases of varicella-zoster pneumonia, complicated with ARDS and MODS, had a favourable outcome.

Key Words: ARDS, Multiple Organ Dysfunction Syndrome, pneumonia, varicella, HIV

\section{INTRODUÇÃO}

A varicela é uma doença exantemática máculo-pápulovesicular, causada pela infecção primária do vírus varicela zoster (VVZ). Geralmente causa epidemias no inverno e na primavera, com maior incidência dos 5 aos 9 anos de idade $^{1,2}$. A transmissão do VVZ ocorre principalmente por meio de gotículas de secreções nasofaríngeas de pessoas infectadas. O período de incubação é de 10 a 25 dias e o período de transmissão ocorre entre 48 horas antes e 
4 dias após o aparecimento do exantema ${ }^{1}$.

Normalmente apresenta curso benigno e autolimitado em crianças $^{3}$, porém a fatalidade é de 10 a 30 vezes maior em adultos ${ }^{1}$, os quais apresentam alta freqüência de complicações, particularmente respiratórias e neurológicas, sendo pneumonia a mais comum². A ocorrência de pneumonia complica o curso da varicela em $5 \%$ a $50 \%$ dos casos adultos ${ }^{2,4}$ e a síndrome da angústia respiratória aguda (SARA) ocorre em cerca de $20 \%$ dos casos de pneumonia por varicela ${ }^{5}$.

O objetivo deste estudo foi apresentar dois casos de pneumonia por varicela que evoluíram com SARA e outras disfunções orgânicas.

\section{RELATO DOS CASOS}

\section{Caso 1}

Paciente do sexo masculino, 15 anos, deu entrada no Pronto Atendimento (PA) em outubro de 2006 com história de varicela havia duas semanas. Apresentava tosse seca, febre, mialgia há 7 dias, sendo diagnosticado pneumonia e tratado com amoxicilina/clavulanato. Horas após a admissão hospitalar, apresentou piora da dispnéia, evoluindo para insuficiência respiratória aguda. Com antecedente de imunocomprometimento pela síndrome da imunodeficiência adquirida (SIDA), relatou que a mãe faleceu por causa desconhecida havia 13 anos. Na admissão na sala de emergência apresentava-se taquidispnéico ( $F R=28 / \mathrm{min})$, taquicárdico ( $\mathrm{FC}=128 \mathrm{bpm}$ ), pressão arterial $(\mathrm{PA})$ : $116 \times 70$ $\mathrm{mmHg}$, Saturação de $\mathrm{O}_{2}\left(\mathrm{SatO}_{2}\right)=88 \%$, escala de coma de Glasgow $=15$ e ausculta pulmonar com crepitações difusas e sibilos. Foi iniciado tratamento terapêutico com aciclovir ( $8 \mathrm{mg} / \mathrm{kg} / \mathrm{dose})$, por via venosa a cada 6 horas, ceftriaxona ( $2 \mathrm{~g} / \mathrm{dia})$, por via venosa, claritromicina (500 $\mathrm{mg}$ ), por via oral a cada 12 horas, associados à corticoterapia com hidrocortisona (200 mg/dia), por via venosa. Devido à piora clínica foi encaminhado para a Unidade de Terapia Intensiva (UTI) antes das primeiras 12 horas de admissão hospitalar.

Na UTI apresentava-se cianótico 2+/4+, febril (38 $\left.{ }^{\circ} \mathrm{C}\right)$, PA: $130 \times 55 \mathrm{mmHg}$, taquicárdico ( $\mathrm{FC}=147 \mathrm{bpm})$, Glasgow 15, sem sinais meníngeos, com lesões pleomórficas crostosas disseminadas, principalmente no tronco, hemodinamicamente estável, sem uso de fármacos vasoativos. Ausculta cardíaca normal e ao exame do aparelho respiratório, encontrava-se taquidispnéico ( $F R=45 / \mathrm{min}$ ), com esforço respiratório e uso da musculatura acessória, ausculta pulmonar com murmúrio vesicular (MV) diminuído nas bases, com estertores crepitantes nos 2/3 inferiores bilateralmente, $\mathrm{SatO}_{2}=71 \%$ com máscara de $\mathrm{O}_{2}(10 \mathrm{~L} / \mathrm{min})$.
Foram realizadas sedação, intubação traqueal e iniciada ventilação mecânica com pressão controlada, fração inspirada de oxigênio $\left(\mathrm{FiO}_{2}\right)$ : 60\%, PEEP: $13 \mathrm{cmH}_{2} \mathrm{O}, \mathrm{FR}: 16$ irpm e pressão inspiratória para manter volume-corrente (VC) de aproximadamente $6 \mathrm{~mL} / \mathrm{Kg}$. O paciente evoluiu com hipotensão arterial não responsiva à reanimação volêmica, sendo introduzido fármaco vasoativo (noradrenalina) e monitorizado com cateter de artéria pulmonar (CAP) e pressão arterial invasiva.

Os exames laboratoriais de admissão encontram-se na tabela 1.

A radiografia de tórax com infiltrado intersticial difuso, em ambos pulmões, cúpulas diafragmáticas livres e a área cardíaca dentro dos limites da normalidade (Figura 1).

Tabela 1 - Exames Laboratoriais da Admissão na UTI (Caso 1)

\begin{tabular}{lc}
\hline \multicolumn{1}{c}{ Exames } & Resultados \\
\hline Glicose & $127 \mathrm{mg} / \mathrm{dL}$ \\
Uréia & $41 \mathrm{mg} / \mathrm{dL}$ \\
Creatinina & $0,8 \mathrm{mg} / \mathrm{dL}$ \\
Leucócitos & $8900 / \mathrm{mm}^{3}$ \\
Plaquetas & $82.000 / \mathrm{mm}^{3}$ \\
Proteína C Reativa (PCR) & $5,87 \mathrm{mg} / \mathrm{dL}$ \\
Transaminase oxalacética (TGO) & $165 \mathrm{U} / \mathrm{L}$ \\
Transaminase pirúvica (TGP) & $133 \mathrm{U} / \mathrm{L}$ \\
pH & 7,34 \\
PaO & (com FiO $\left.{ }_{2} 100 \%\right)$ \\
PaCO & \\
$\mathrm{HCO}_{3}$ & $93 \mathrm{mmHg}$ \\
Excesso de base & $42 \mathrm{mmHg}$ \\
Saturação de $\mathrm{O}_{2}$ & 22 \\
Lactato arterial $_{\text {Sorologia para HIV (Elisa) }}$ & -3 \\
Carga viral & $97 \%$ \\
$\mathrm{CD}$ & $3,3 \mathrm{mEq} / \mathrm{L}$ \\
\hline
\end{tabular}

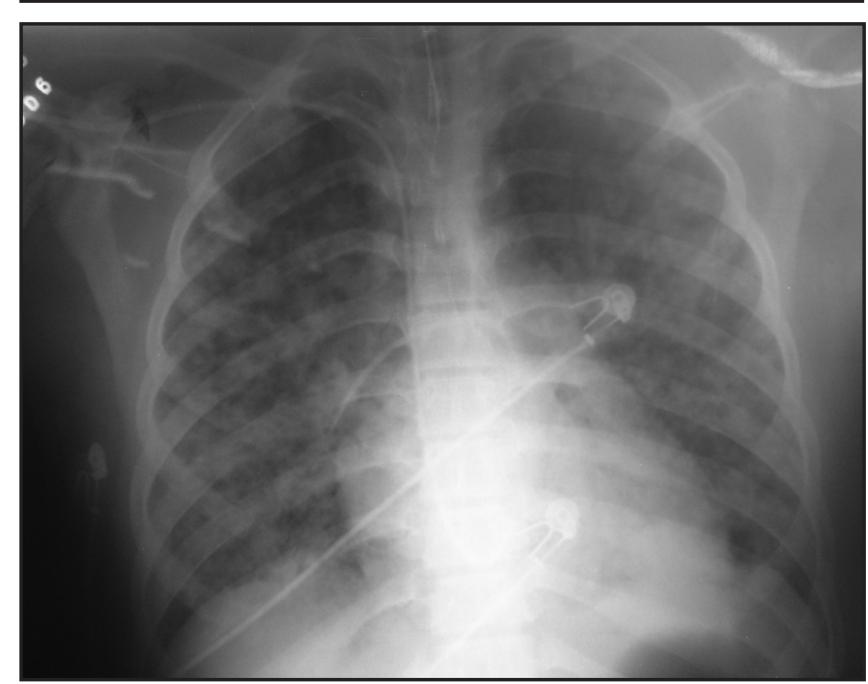

Figura 1 - Radiografia de Tórax após Intubação Traqueal e Monitorização com Cateter de Artéria Pulmonar. 
Os valores das variáveis obtidas com o CAP foram: índice cardíaco $(\mathrm{IC})=4,17 \mathrm{~L} / \mathrm{min} \cdot \mathrm{m}^{2}$, pressão de oclusão da artéria pulmonar (POAP): 14 mmHg, índice de resistência vascular sistêmica $($ IRVS $)=1341$ $\mathrm{Ds} / \mathrm{cm}^{5} / \mathrm{m}^{2}$, índice de resistência vascular pulmonar $($ IRVP $)=383 \mathrm{Ds} / \mathrm{cm}^{5} / \mathrm{m}^{2}$, oferta de oxigênio $\left(\mathrm{DO}_{2}\right)=$ $793 \mathrm{~mL} / \mathrm{min} / \mathrm{m}^{2}$, consumo de oxigênio $\left(\mathrm{VO}_{2}\right)=215$ $\mathrm{mL} / \mathrm{min} \cdot \mathrm{m}^{2}$, taxa de extração de oxigênio $=27 \%$ e lactato sérico $=1,6 \mathrm{mEql} / \mathrm{L}$

Evoluiu com melhora progressiva das disfunções orgânicas (hematológica e cardiovascular) até o $4^{\circ}$ dia de internação, com declínio progressivo dos níveis séricos de proteína C-reativa e da contagem de leucócitos, ausência de febre após 48 horas do início do uso de antimicrobianos e aumento da relação $\mathrm{PaO}_{2} / \mathrm{FiO}_{2}$, sendo extubado após 5 dias de ventilação mecânica. Transferido para a enfermaria após 9 dias na UTI. Recebeu alta hospitalar após 2 dias na enfermaria.

\section{Caso 2}

Paciente do sexo feminino, 29 anos, deu entrada na sala de emergência em outubro de 2006 com lesões cutâneas vésico-bolhosas disseminadas e história de febre, mialgia e adinamia havia 5 dias. Há um dia iniciou com tosse seca e dispnéia, com piora da falta de ar nas últimas 6 horas. Antecedentes pessoais: gastrite há 5 meses, com melhora após tratamento clínico. Antecedentes familiares: filho com diagnóstico e acompanhamento de varicela há aproximadamente 10 dias. $\mathrm{Na}$ admissão na sala de emergência apresentava taquicardia (FC = $140 \mathrm{bpm}$ ), PA: $110 \times 70 \mathrm{mmHg}$, taquidispnéia (FR $=52 / \mathrm{min})$, cianose $(++/ 4+)$, SatO $2=70 \%$, esfor ço respiratório com uso da musculatura acessória, ausculta pulmonar com crepitações difusas e com agitação psicomotora. A paciente foi submetida à intubação orotraqueal, com dificuldade técnica, associada à presença de vômitos e broncoaspiração. Foi iniciado tratamento terapêutico com aciclovir $(10 \mathrm{mg} / \mathrm{kg} / \mathrm{dose}$ ) a cada 8 horas, ceftriaxona (2 g/dia), metilprednisolona ( $1 \mathrm{mg} / \mathrm{kg})$ a cada 8 horas, todos por via venosa e encaminhada para UTI.

Os exames laboratoriais de admissão encontramse na tabela 2.

A radiografia de tórax apresentava infiltrado intersticial pulmonar predominantemente em bases, bilateral (Figura 2).
Tabela 2 - Exames Laboratoriais da Admissão na UTI (Caso 2)

\begin{tabular}{lc}
\hline Exames & Resultados \\
\hline Glicose & $106 \mathrm{mg} / \mathrm{dL}$ \\
Uréia & $75 \mathrm{mg} / \mathrm{dL}$ \\
Creatinina & $2,1 \mathrm{mg} / \mathrm{dL}$ \\
Leucócitos & $6800 / \mathrm{mm}^{3}$ \\
Plaquetas & $101.000 / \mathrm{mm}^{3}$ \\
Hematócrito $(\mathrm{Ht})$ & $51 \%$ \\
Hemoglobina (Hb) & $17,1 \mathrm{~g} / \mathrm{dL}$ \\
Proteína C-reativa (PCR) & $28,2 \mathrm{mg} / \mathrm{dL}$ \\
Transaminase oxalacética (TGO) & $220 \mathrm{U} / \mathrm{L}$ \\
Transaminase pirúvica (TGP) & $45 \mathrm{U} / \mathrm{L}$ \\
pH & 7,25 \\
PaO & (com FiO $\left.{ }_{2} 100 \%\right)$ \\
PaCO $_{2}$ & $107 \mathrm{mmHg}$ \\
$\mathrm{HCO}_{3}$ & $41 \mathrm{mmHg}$ \\
Excesso de base $_{\text {Saturação de } \mathrm{O}_{2}}$ & 18 \\
Lactato arterial $_{\text {Sorologia para HIV (Elisa) }}$ & -9 \\
\hline
\end{tabular}

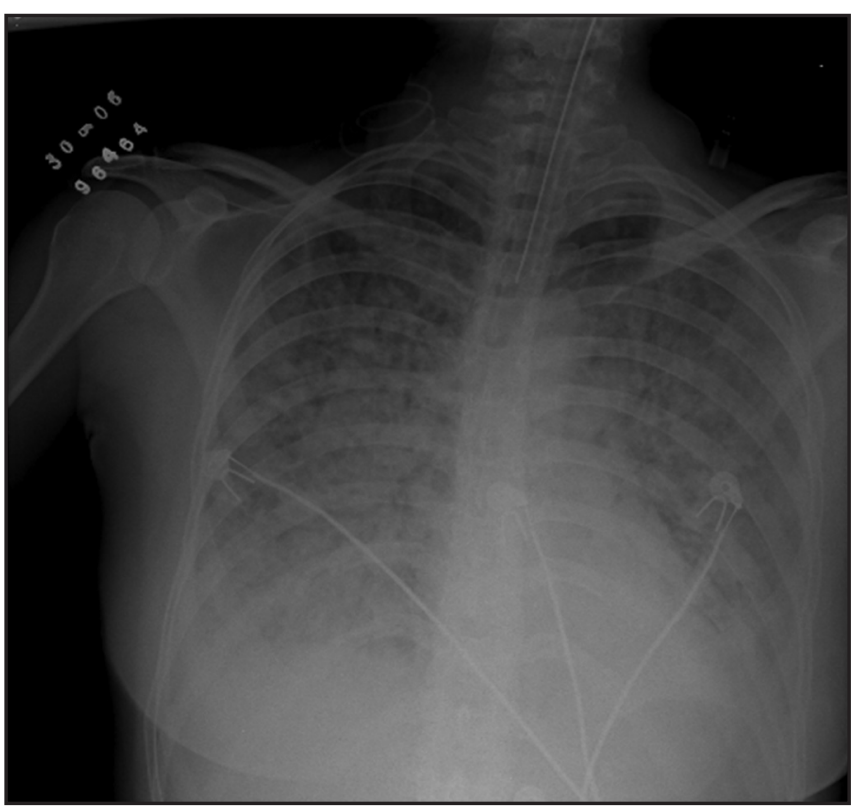

Figura 2 - Radiografia de Tórax da Admissão na UTI.

Após a admissão na UTI, a paciente apresentava-se taquicárdica ( $\mathrm{FC}=130 \mathrm{bpm}$ ), desidratada, com palidez cutâneo-mucosa +/4+, Glasgow estimado: 15, sem sinais meníngeos, com lesões cutâneo-mucosas pleomórficas (vesículas e crostas disseminadas na face, tronco e membros), débito urinário $>0,5 \mathrm{~mL} / \mathrm{kg} / \mathrm{h}$, com tubo traqueal, em ventilação mecânica (modo: volume controlado, $\mathrm{FiO}_{2}$ : 100\%, VC: $6 \mathrm{~mL} / \mathrm{kg}$, PEEP: 10 $\mathrm{cmH}_{2} \mathrm{O}, \mathrm{FR}: 16 \mathrm{irpm}$, fluxo: $\left.60 \mathrm{~L} / \mathrm{min}\right)$, com SatO ${ }_{2}: 96 \%$, 
apresentando crepitações difusas na ausculta pulmonar e roncos esparsos.

Após a implantação de acesso venoso central e reanimação volêmica guiada por pressão venosa central (PVC), saturação venosa central de $\mathrm{O}_{2}\left(\mathrm{SvcO}_{2}\right)$ e lactato, a paciente evoluiu com melhora da perfusão tecidual evidenciada pela melhora da $\mathrm{SvcO}_{2}$ de $69 \%$ para $76 \%$ e lactato de $7,6 \mathrm{mEq} / \mathrm{L}$ para $1,2 \mathrm{mEq} / \mathrm{L}$ em 24 horas. Apresentou também diminuição dos valores de creatinina $(2,1 \mathrm{mg} / \mathrm{dL}$ para $0,8 \mathrm{mg} / \mathrm{dL})$.

O modo ventilatório foi modificado para pressão controlada, PEEP: $15 \mathrm{cmH}_{2} \mathrm{O}$, pressão Inspiratória para manter o volume-corrente próximo de $6 \mathrm{~mL} / \mathrm{kg}$ e foi realizado recrutamento alveolar. Após recrutamento alveolar foi mantida ventilação mecânica protetora (VC = $6 \mathrm{~mL} / \mathrm{kg}$ e pressão de platô $<30 \mathrm{cmH}_{2} \mathrm{O}$ ).

Evoluiu com reversão das disfunções orgânicas (hematológica e metabólica) após o $4^{\circ}$ dia de internação, diminuição progressiva dos níveis de PCR, o qual se normalizou após $\circ 6^{\circ}$ dia, normalização da relação $\mathrm{PaO}_{2} / \mathrm{FiO}_{2}$, sendo extubada após 14 dias de ventilação mecânica. Recebeu alta da UTI após 18 dias de internação, com todas as disfunções orgânicas revertidas e com tetraparesia discreta a moderada.

\section{DISCUSSÃO}

A pneumonia por varicela é a complicação mais comum de infecção primária em adultos ${ }^{6}$. Os fatores de risco para o desenvolvimento de pneumonia em adultos com varicela primária incluem tabagismo, gravidez e estado imunológico comprometido ${ }^{7-10}$. Algum tipo de imunodepressão está presente em $12 \%$ a $33 \%$ dos casos de pneumonia por varicela ${ }^{9,10}$, associando-se a elevada morbimortalidade. A mortalidade é estimada em $10 \%$ a $30 \%$ em indivíduos imunocompetentes e aumenta para $50 \%$ em pacientes imunodeprimidos ${ }^{8}$.

Dentre os pacientes com pneumonia por varicela que necessitaram de cuidados em UTI, a incidência de SARA variou de $16 \%$ a $37 \%^{7,11,12}$.

Foram apresentados dois casos de pneumonia por varicela que cursaram com SARA e disfunções orgânicas múltiplas, internados no ano de 2006 em UTI com 44 leitos de um hospital terciário.

No primeiro caso, o paciente tinha 15 anos e era imunocomprometido pela SIDA. No segundo caso, a doença ocorreu em uma paciente de 29 anos, imunocompetente, que teve sua evolução complicada por broncopneumonia aspirativa. Ambos foram tratados com antibioticoterapia de amplo espectro além do aciclovir e corticosteróides, pois no primeiro caso suspeitou-se inicialmente de pneumonia comunitária atípica, e no segundo havia pneumonia aspirativa associada.

Os dois pacientes apresentaram disfunções orgânicas múltiplas, sendo que as disfunções respiratória, hematológica e metabólica ocorreram nos dois casos $^{13}$. O primeiro caso apresentou ainda disfunção cardiovascular e o segundo caso disfunção renal. Todas foram revertidas até a alta da UTI.

Plaquetopenia e elevação de transaminases, alterações laboratoriais comuns em casos de varicela complicada, ocorreram nos dois casos 6-7,9,14-18.

Os dois pacientes relatados apresentaram radiografia de tórax com infiltrado intersticial bilateral, compatíveis com o padrão radiográfico comumente encontrado em casos de pneumonia por varicela: infiltrado pulmonar intersticial ou nodular predominantemente em bases ${ }^{19-20}$. A pneumonia por varicela complicada com a SARA é complicação grave e relativamente rara. $\mathrm{O}$ tratamento com aciclovir e corticosteróides determina melhores resultados quando iniciado precocemente ${ }^{18-22}$. Outras complicações descritas da varicela incluem coagulação intravascular disseminada (CIVD), hepatite, encefalite e miocardite ${ }^{18,23-25}$.

Evidências recentes sugerem que a vacinação para varicela é efetiva na prevenção ou na modificação da gravidade da infecção por varicela se utilizada em menos de 3 dias e possivelmente até 5 dias após a exposição ao vírus ${ }^{19}$.

A provável recomendação para o uso da imunoglobulina intravenosa seria para pacientes imunossuprimidos e gestantes que tiveram contato com uma fonte confirmada de varicela e deve ser utilizada com um tempo menor de 96 horas após a exposição para sua efetivade máxima ${ }^{19}$.

Diante do exposto, os dois casos de pneumonia por varicela, que apresentaram SARA e disfunções de múltiplos órgãos, obtiveram boa resposta ao tratamento farmacológico e à ventilação mecânica protetora.

\section{REFERÊNCIAS}

01. Szpeiter N, Toledo PVM, Carvalho MTM et al - Varicela e Herpes Zoster, em: Lopes AC, Amato VC - Tratado de Clínica Médica. São Paulo: Roca; 2006;3854-3858.

02. Jones AM, Thomas N, Wilkins EG - Outcome of varicella pneumonitis in immunocompetent adults requiring treatment in a high dependency unit. J Infect, 2001;43:135-139.

03. Somekh E, Maharashak N, Shapira $Y$ et al - Hospitalization for primary varicella-zoster virus infection and its complications in patients from Southern Israel. Infection, 2000;28:200-204.

04. Gasparetto EL, Warszawiak D, Tazoniero P et al - Varicella pneumonia in immunocompetent adults: report of two cases, with emphasis on high-resolu- 
tion computed tomography findings. Braz J Infect Dis, 2005;9:262-265.

05. von Mach MA, Kaes J, Omogbehin B et al - An update on interventional lung assist devices and their role in acute respiratory distress syndrome. Lung, 2006;184:169-175.

06. Chou DW, Lee $\mathrm{CH}$, Chen CW et al - Varicella pneumonia complicated by acute respiratory distress syndrome in an adult. J Formos Med Assoc, 1999;98:778-782.

07. Martinez Segura JM, Gutierrez Oliva A, Maravi Poma E et al - Severe chickenpox pneumonia. Rev Clin Esp 2003;203:591-594.

08. Conti JA, Karetzky MS - Varicella pneumonia in the adult. N J Med, 1989;86:475-478.

09. Paytubi C, Negredo E, Ferrer S et al - Varicella pneumonia in the adult. Study of 9 cases. An Med Interna, 2001;18:312-316.

10. Aleman Llanso C, Garcia Quintana AM, Alegre Martin J et al - Varicella pneumonia in the adult. A review of 25 cases. Rev Clin Esp, 1997;197:690-692.

11. Ho BC, Tai DY - Severe adult chickenpox infection requiring intensive care. Ann Acad Med Singapore, 2004;33:84-88.

12. Hockberger RS, Rothstein RJ - Varicella pneumonia in adults: a spectrum of disease. Ann Emerg Med, 1986;15:931-934.

13. Vincent $\mathrm{JL}$ - Organ dysfunction as an outcome measure: the SOFA score. Sepsis, 1997;1:53-54

14. Hepburn NC, Carley RH - Adult respiratory distress syndrome secondary to varicella infection in a young adult. J R Army Med Corps, 1989;135:8183.

15. Rodriguez Borregan JC, Dominguez Artiga MJ, Minambres E et al - Varicella pneumonia in adults: 30 cases. An Med Interna, 2003;20:612-616.
16. El-Daher N, Magnussen R, Betts RF - Varicella pneumonitis: clinical presentation and experience with acyclovir treatment in immunocompetents adults. Int J Infect Dis, 1998;2:147-151.

17. Shirai T, Sano K, Matsuyama S et al - Varicella pneumonia in a healthy adult presenting with severe respiratory failure. Intern Med, 1996;35:315318.

18. Lee $\mathrm{S}$, Ito $\mathrm{N}$, Inagaki $\mathrm{T}$ et al - Fulminant varicella infection complicated with acute respiratory distress syndrome, and disseminated intravascular coagulation in an immunocompetent young adult. Intern Med, 2004;43:1205-1209.

19. Mohsen $\mathrm{AH}$, McKendrick $\mathrm{M}$ - Varicella pneumonia in adults. Eur Respir J, 2003; 21:886-891.

20. Potgieter PD, Hammond JM - Intensive care management of varicella pneumonia. Respir Med, 1997; 91:207-212.

21. Bautista Rodriguez MD, Bravo-Rodriguez F de A, Fuentes Jimenez F et al - Glucocorticoids and immunoglobulins in an acute respiratory syndrome secondary to varicella pneumonia. Med Clin, 2004;122:238-238.

22. Mer M, Richards GA - Corticosteroids in life-threatening varicella pneumonia. Chest, 1998;114:426-431.

23. Ross JS, Fanning WL, Beautyman $W$ et al - Fatal massive hepatic necrosis from varicella-zoster hepatitis. Am J Gastroenterol, 1980;74:423427.

24. Koskiniemi M, Piiparinen $\mathrm{H}$, Rantalaiho $\mathrm{T}$ et al - Acute central nervous system complications in varicella zoster virus infections. J Clin Virol, 2002;25:293-301.

25. Osama SM, Krishnamurti S, Gupta DN - Incidence of myocarditis in varicella. Indian Heart J, 1979;31:315-320. 